\title{
LES CRISES DE LA CONSTRUCTION EUROPEENNE
}

\author{
Olivier BLIN
}

Maître de Conférences HDR, Université Toulouse 1 Capitole, IRDEIC

Il était tentant de commencer cette contribution par la fameuse rime musicale " $M a$ petite entreprise, Connaît pas la crise" imaginée par Alain Bashung à la toute fin des années 1990 ; oui, mais voilà... appliquée à la construction européenne, la formule -qui dira certainement quelque chose aux amateurs de musique française de plus de 35 ans-, ne convient pas : tout simplement parce que la "grande entreprise" (européenne) connaît remarquablement la crise !

En premier lieu, il n'est pas inutile de rappeler -car on focalise le plus souvent sur les objectifs économiques de l'Europe, les plus évidents- que l'idée de crise est consubstantielle à la construction européenne, puisqu'elle a été lancée au début des années 1950 après un conflit armé de dimension mondiale particulièrement mortifère et avec l'objectif d'éviter qu'un tel épisode paroxystique ne se reproduise sur le Vieux continent (d'où le choix, au départ, de la mise en commun du charbon et de l'acier, deux produits stratégiques en cas de conflit armé, dans le cadre de la $\mathrm{CECA}^{1}$ ).

En deuxième lieu, le moins que l'on puisse dire c'est que la construction européenne a connu son lot de crises, au point que certaines mauvaises langues vont jusqu'à prétendre que l'expression "crise européenne" constituerait un pléonasme... Sans aller jusque là, il faut bien reconnaître que les crises européennes ont été nombreuses, parmi lesquelles : l'échec de la Communauté européenne de défense (CED, 1954), la crise de la chaise vide (1965), la crise budgétaire des années 1980, les crises alimentaires des années 1990 (avec l'affaire de la "vache folle"), la crise institutionnelle de 1999 (avec la démission de la Commission dirigée par Jacques Santer), la crise constitutionnelle de 2005 (après l'échec puis l'abandon de la Constitution européenne), la crise actuelle de la zone euro... Et

\footnotetext{
${ }^{1}$ Traité de Paris du 18 avril 1951 instituant la Communauté européenne du Charbon et de l'acier ; prévue pour 50 ans à partir de son entrée en vigueur (1952), la CECA a disparu en tant que telle en 2002, dans une indifférence générale que son rôle pionnier aurait pourtant dû lui épargner. Sur les conditions de sa disparition, voy. P. DAILLIER, "La disparition de la CECA le 23 juillet 2002 - Des problèmes de succession d'organisations internationales", Mélanges J.-C. GAUTRON, Paris, Pedone, 2004, pp. 19-28.
} 
les observateurs comme les opinions publiques se sont progressivement habitués à cette succession de crises et relances en Europe.

En troisième et dernier lieu, rappelons que l'Union européenne connaît depuis 2008 une crise sans précédent, au regard de son intensité, de son caractère protéiforme et de sa durée ; au point que diverses analyses politico-juridiques se sont intéressées non seulement aux causes et manifestations de ces crises mais également aux scenarii de sortie de $\operatorname{crise}^{2} \ldots$

Bien que présente dans n'importe quel système social, juridique, la crise se manifestement donc particulièrement souvent dans le cadre européen donnant ainsi raison à Jean Monnet qui affirmait dans ses Mémoires : 'J'ai toujours pensé que l'Europe se ferait dans la crise et qu'elle serait la solution donnée à ces crises ”. D'ailleurs, l'analyse de la construction européenne "dans le temps long” pour reprendre l'expression du Professeur Quermonne ${ }^{4}$ - permet de constater que le phénomène de crise constitue l'une des constantes du projet européen, ou, pour le dire autrement, que “(...) la crise est inscrite dans les gènes de l'Union européenne, dans son $A D N$ ".5.

Justement, il nous faut maintenant préciser le sujet retenu : on entendra le terme "crise" plutôt dans son sens grec initial (décision importante, choix, jugement) renvoyant à l'idée d'un moment-clé, d'un moment où tout se décide, plutôt que dans son acception moderne où la crise est synonyme de catastrophe, de chaos voire de rupture : cette approche initiale semble particulièrement correspondre à la réalité européenne comme nous le reverrons. Ensuite, il s'agira des crises de la construction européenne, c'est-à-dire celles qui sont intimement liées à la création puis au développement des Communautés et de l'Union européennes, et non pas de toute crise à l'échelle du continent européen (on écartera ainsi la chute du modèle soviétique ou la guerre dans l'ex-Yougoslavie dans les années 1990). Il ne sera pas plus question de l'action européenne dans la gestion des crises, qu'elles soient internes ${ }^{6}$ comme internationales $^{7}$. Enfin, dans la mesure où cette contribution prend place dans la thématique consacrée au "Droit issu de la crise", nous nous intéresserons tout naturellement aux solutions qui ont pu être dégagées dans le cadre

\footnotetext{
${ }^{2}$ C. Blumann et F. Picod, L’Union européenne et les crises, Bruxelles, Bruylant, coll. Droit de l’Union européenne, 2011 ; F. Kinsky, "L'Europe est-elle sortie de la crise ?", Mélanges Touscoz, Nice, France Europe Editions, 2007, pp. 544-555 ; P. Magnette et A. Weyembergh, L'Union européenne : la fin d'une crise?, Bruxelles, Editions de l'Université de Bruxelles, 2008.

${ }^{3}$ Mémoires, Paris, Fayard, 1976, p. 488.

${ }^{4}$ J.-L. Quermonne, L'Union européenne dans le temps long, Paris, Presses de Sciences-Po, 2008.

${ }^{5}$ C. Blumann et F. Picod, "Rapport introductif général", in L'Union européenne et les crises, op. cit. p. 1.

${ }^{6}$ Voy. la contribution de F. CAULET dans cet ouvrage.

${ }^{7}$ Voy. C. Blumann et F. Picod, op. cit., pp. 167-291 et B. Delcourt, "La gestion des crises : un nouvel élan pour l'Europe ? Analyse de l'implication de l'UE au Kosovo", in L'Union européenne : la fin d'une crise?, op. cit., pp.103-114.
}

\section{Crise(s) et droit}


européen pour résoudre ces crises ; loin d'êtres fatales, les crises européennes ont en effet souvent permis un renouveau, une relance du projet européen ${ }^{8}$.

Dès lors, qu'est-ce qui caractérise les crises de la construction européenne ? Sont-elles forcément différentes de celles qui surgissent dans d'autres systèmes ou contextes juridiques?

A la réflexion, il nous semble que ces crises ont évolué dans leur nature au cours du temps, l'actuelle crise de sens que connaît l'Union succédant à de nombreuses crises de croissance (I) ; par ailleurs, les réponses apportées à ces crises ont systématiquement permis un "rebond" du projet européen, participant ainsi à son dynamisme (II).

\section{I - DES CRISES DE CROISSANCE À UNE CRISE DU SENS}

En préalable, il convient de noter que la cause des crises européennes est, dans la plupart des cas, de nature endogène : il s'agira pour l'essentiel de problèmes de fonctionnement institutionnel ou de divergences politiques entre Etats membres ; tout juste peuton mentionner comme crise européenne d'origine exogène, l'actuelle crise financière qui a débuté par la faillite (en juillet 2007) du marché hypothécaire américain et qui a dégénéré aujourd'hui en une crise de la dette des Etats et en une crise de l'Euro'.

Ceciétant dit, il apparaît que les crises européennes ont été pendant longtemps des crises d'évolution, de croissance, qui ont perturbé ponctuellement le cours des Communautés et de l'Union, mais sans que la philosophie générale du système ou ses principales finalités ne soient véritablement remises en cause (A) ; depuis quelques années, la crise frappant l'Union européenne est plus profonde en ce qu'elle touche aux fondements même du projet européen et se trouve dès lors susceptible d'hypothéquer réellement son avenir (B).

\section{A - Les multiples crises de croissance}

Dans la logique fonctionnaliste des concepteurs des Communautés, la construction européenne a été conçue comme un processus continu ; dès lors, il est assez naturel que celle-ci ait connu divers blocages, lesquels ont été à chaque fois surmontés, avec, évidemment, des résultats plus ou moins satisfaisants pour le juriste.

Les principales crises ont été institutionnelles ou politiques, étant entendu que la distinction est forcément réductrice puisque la plupart des crises appartiennent finalement à l'une et à l'autre des catégories.

* Les crises de nature institutionnelle sont certainement les plus attendues, les plus évidentes, au sein d'un système complexe -bien que réduit au départ au fameux "quadripartisme institutionnel"- reposant sur un équilibre toujours instable entre des légitimités

${ }^{8}$ Construction européenne : crises et relances, Paris, Economica, 2009.

9 Voy. A. Buzelay, "Les sources des crises monétaires et financières en Europe", in L'Union européenne et les crises, op. cit., pp. 113-120.

\section{Crise(s) et droit}


différentes (intégrative, intergouvernementale, démocratique et juridictionnelle) tenues de collaborer pour l'intérêt commun ${ }^{10}$.

D'intensité variable, ces crises institutionnelles ont été relativement nombreuses, surtout à partir des années 1980 lorsque le Parlement européen a commencé à vouloir étendre son influence au sein du "triangle institutionnel". Deux exemples permettent d'illustrer cette réalité : la crise budgétaire qui a marqué les années 1980 d'abord, et la démission de la Commission Santer à la fin des années 1990 ensuite.

Il faut se souvenir que pendant longtemps -plus précisément jusqu'au traité de Maastricht de 1992- les prérogatives du Parlement européen se cantonnaient pour l'essentiel au domaine budgétaire, prérogatives d'ailleurs significativement renforcées par les traités (budgétaires) du 22 avril 1970 et 22 juillet 1975. De sorte que la procédure en résultant consacrait le Parlement européen comme co-détenteur du pouvoir budgétaire avec le Conseil des ministres ${ }^{11}$, l'intervention de la Commission se réduisant quant à elle à la transmission du projet de budget.

Le moins que l'on puisse dire c'est que le Parlement européen a exercé avec beaucoup d'attention son pouvoir budgétaire ${ }^{12}, n^{\prime}$ hésitant pas à l'utiliser -à une époque où il n'avait quasiment pas de rôle législatif- pour faire pression sur les Etats ; à telle enseigne qu'il a mené pendant plusieurs années une véritable "guérilla budgétaire" contre le Conseil des ministres allant jusqu'à provoquer une situation de vive tension en rejetant globalement le budget en 1979, puis à nouveau en 1988.

Le Parlement européen a également misé sur le rapport de force afin de faire évoluer l'équilibre institutionnel à son avantage dans ses rapports avec la Commission européenne cette fois, à la fin des années 1990.

Plus précisément, à la suite de rumeurs d'erreurs de gestion, de pratiques frauduleuses et de népotisme se répandant à partir de l'été 1998 et visant plusieurs membres de la Commission dirigée par le Luxembourgeois Jacques Santer, le Parlement européen décida, début 1999, de mettre en place un "Comité des sages" chargé de faire toute la lumière sur ces graves allégations. Le constat effectué par celui-ci dans son rapport remis aux parlementaires le 15 mars 1999 fut accablant non seulement pour les personnes visées -parmi lesquelles Mme Edith Cresson, ancien premier ministre français et à l'époque

\footnotetext{
${ }^{10}$ Sur le schéma institutionnel initial et son évolution, voy. spéc. : P. Pescatore, "L’exécutif communautaire : justification du quadripartisme institué par les traités de Paris et Rome", $C D E$ 1978, pp. 387-406.

${ }^{11}$ Avec le traité de Lisbonne, le Parlement devient véritablement l'égal du Conseil des ministres dans le cadre de la procédure budgétaire qui prend la forme d'une "procédure de co-décision simplifiée" : J.-P. JACQUE, Droit institutionnel de l'Union européenne, Paris, Dalloz, coll. Cours, 2010, p. 269.

${ }^{12}$ Ibid.

${ }^{13}$ R. MeHDI, “Les crises institutionnelles”, in L’Union européenne et les crises, op. cit., pp. 121-141.
}

\section{Crise(s) et droit}


Commissaire européen à la recherche ${ }^{14}$ - d'un point de vue moral et surtout juridique, mais également pour la Commission en tant qu'institution, coupable de dysfonctionnements manifestes. Plutôt que d'attendre l'adoption d'une motion de censure à son encontre -à laquelle cette Commission avait d'ailleurs échappé de justesse en janvier $1999^{15}$-, le Président de la Commission présenta dès le lendemain de la remise dudit rapport la démission de l'ensemble du collège ${ }^{16}$.

On l'aura compris, même si la Commission n'a pas été juridiquement renversée par le Parlement, elle a été contrainte politiquement à la démission à la suite de ce rapport; dès lors, l'équilibre entre les deux institutions s'en est trouvé modifié en faveur du Parlement, ce dernier n'hésitant pas par la suite à brandir cette "épée de Damoclès" que constitue désormais la censure -pourtant hypothèse d'école jusqu'en 1999- afin d'obtenir certains engagements précis à son endroit.

* Les crises politiques ont aussi jalonné l'évolution de la construction européenne, alternant avec les précédentes.

Parmi les crises de nature politique, nous en retiendrons trois qui impliquent systématiquement la France : celle de la CED, celle de la "chaise vide" et celle ouverte par l'échec de la Constitution européenne ${ }^{17}$.

L'épisode de l'échec de la Communauté européenne de défense (CED) constitue chronologiquement la première véritable crise de la construction européenne ${ }^{18}$.

La France était à l'origine du projet de CED -c'est-à-dire la création d'une armée européenne- signé, malgré des résistances, par les six Etats fondateurs le 27 mai 1952. Pourtant, le 30 août 1954 et alors que quatre partenaires sur cinq avaient ratifié le texte, l'Assemblée nationale française le rejetait, écartant du même coup pour une longue période toute velléité d'Europe politique à travers l'abandon corollaire de la Communauté Politique européenne (CPE). Même si cet échec n'était pas totalement imprévisible -la version définitive du texte avait fait l'objet d'un compromis laborieux d'une part et les débats avaient été vifs au sein de la classe politique de chacun des Etats membres d'autre

\footnotetext{
${ }^{14}$ Pour Mme Cresson, l'affaire ne s'est pas arrêtée à la démission de la Commission à laquelle elle appartenait ; sept années plus tard, elle a été personnellement condamnée pour violation de ses obligations statutaires : CJCE, 11 juillet 2006, Commission c/ Edith Cresson, aff. C-432/04, Rec. I-6387.

${ }^{15}$ Il est en effet prévu depuis 1957 que la motion de censure recueille une double majorité (majorité des membres du Parlement européen et des deux tiers des suffrages exprimés) pour être adoptée.

${ }^{16}$ Pour des précisions, A. Sevens, "La chute de la Commission Santer", RFAP 2000, vol. 95, pp. 369-379.

${ }^{17}$ Il y en a bien sûr d'autres, notamment la "crise autrichienne" de 1999 suite à l'arrivée à la tête du gouvernement du chef de file du parti conservateur Jörg Haïder ; pour des précisions, voy. F. Fines, "De l'influence de la crise autrichienne sur l'avenir de l'Europe", Mélanges Isaac, Toulouse, Presses de l'Université des Sciences sociales de Toulouse, 2004, pp. 105-120.

${ }_{18}$ Pour des détails, C. Pineau et C. Rimbaud, Le grand pari. L'aventure du traité de Rome, Paris, Fayard, 1991, spéc. pp. 103-150.
}

\section{Crise(s) et droit}


part-, il constituait incontestablement une crise grave ${ }^{19}$, certains observateurs allant même jusqu'à considérer qu'il sonnait le glas de la construction européenne. Pourtant, le mouvement sera relancé de manière décisive quelques mois plus tard avec, in fine, la création des deux autres Communautés ${ }^{20}$.

De même, peu après le premier refus de l'élargissement des Communautés européennes au Royaume-Uni -il y en aura un second en 1967 avant son adhésion en 1973-, De Gaulle sera à l'origine de la "crise de la chaise vide".

A partir de juillet 1965, celui-ci décide en effet de ne plus envoyer de représentants au Conseil des ministres des Communautés pour protester contre les modalités de financement de la Politique agricole commune (PAC) et surtout la généralisation du vote à la majorité qualifiée (au détriment de l'unanimité), notamment en matière agricole. Chacun le sait, le "Compromis de Luxembourg" de janvier 1966 permettra une "sortie de crise" satisfaisante pour la France d'un côté et pour ses cinq partenaires de l'autre, mais conduira de facto à la pratique du consensus au Conseil jusqu'au milieu des années 1980.

Enfin, il y a quelques années, la France a de nouveau été à l'origine d'un blocage important du processus d'approfondissement européen. Durant la phase de ratification du traité instituant une Constitution pour l'Europe (ou “Constitution européenne"), signé officiellement le 29 octobre 2004, les Français d'abord (29 mai 2005) ${ }^{21}$ et les Néerlandais ensuite $\left(1^{\text {er }} \text { juin 2005) }\right)^{22}$ ont repoussé le texte par référendum. Comme en 1992 (les Danois refusant le traité de Maastricht) et en 2001 (les Irlandais votant contre le traité de Nice), un traité européen était remis en cause par des citoyens de l'Union s'exprimant directement. Malgré un nombre important de ratifications obtenues, avant mais également après ces référendums négatifs, l'entrée en vigueur de la Constitution européenne était juridiquement bloquée. Le coup a été extrêmement rude pour les tenants de la poursuite de l'intégration européenne, à l'instar de M. Jacques Delors, ancien président de la Commission européenne, qui a pu déclarer qu'il s'agissait assurément là de la crise la plus grave de l'histoire européenne...

A cette occasion, la question a pu être posée de savoir si cet échec de la "Constitution européenne" était la cause ou le révélateur de la crise de l'Union ${ }^{23}$. A notre sens, la réponse ne fait guère de doute ; l'échec constitutionnel constitue bien le révélateur d'une crise, diffuse et relativement ancienne, en un mot une crise de sens qui touche le cœur même du projet européen.

\footnotetext{
${ }^{19}$ Voy. M.-T. Biтsch, "Crises et relances de la construction européenne, des années 50 aux années 80", in Construction européenne : crises et relances, op. cit., pp. 7-26, spéc. pp. 9-10.

${ }^{20}$ Voy. infra II (B).

${ }^{21} 54,68 \%$ "contre" et 45,32\% "pour", avec une participation de 69,34\% des électeurs.

${ }^{22} 61,5 \%$ "contre" et 38,5\% "pour", avec une participation de 63,3\% des électeurs.

${ }^{23}$ R. Kovar, "L'échec de la Constitution pour l'Europe : cause ou révélateur de la crise de l'Union européenne? », Mélanges Touscoz, op. cit., pp. 514-543.
}

\section{Crise(s) et droit}




\section{B - L'actuelle crise de sens}

Les précédentes crises, qu'elles soient politiques ou institutionnelles, avaient fondamentalement un caractère conjoncturel et pouvaient difficilement être interprétées comme un désaveu de la logique caractérisant la construction européenne dès l'origine. Depuis quelques années, le mal est plus profond, concrétisant une lente et manifeste dégradation de l'idée européenne ; il conduit à s'interroger sérieusement sur la direction prise par l'Union : Quo Vadis Europa?

Dès le départ, la construction européenne comportait une finalité politique ; le processus pouvait se résumer ainsi : l'institutionnel d'abord, l'économique ensuite, et le politique enfin.

Le cadre institutionnel -innovant et performant ${ }^{24}$ - une fois mis en place et régulièrement enrichi, la priorité a été donnée au Marché commun, puis unique, des années 1960 aux années 1990, lequel devait déboucher sur la réalisation de l'Union économique et monétaire (UEM). A la toute fin des années 1980, l'engagement de l'Allemagne à demeurer dans l'Europe après la réunification et à adopter la future monnaie commune fut lié à la nécessité d'une refondation politique du projet européen ${ }^{25}$. Telle était donc l'ambition du traité de Maastricht créant l'Union européenne qui, s'il procédait incontestablement à un approfondissement, marquait parallèlement l'affirmation de l'inter-gouvernementalisme à travers la création des deuxième et troisième piliers de l'Union (relatifs respectivement à la Politique étrangère et de sécurité commune (PESC)) et à la Coopération en matière de justice et d'affaires intérieures (CJAI) devenue Coopération policière et judiciaire en matière pénale (CPJP) à partir d'Amsterdam $)^{26}$.

$\mathrm{Au}$ caractère peu lisible de la nouvelle architecture, s'est ajoutée une succession de traités européens (à un rythme de plus en plus soutenu dans la période 1992-2004 ${ }^{27}$ ) ne comportant que des dispositions techniques, sans aucun renforcement concomitant de l'Union politique.

Par conséquent, alors que les Etats n'arrivaient pas à se mettre d'accord sur le sens à donner à l'Europe, les opinions publiques ont eu logiquement du mal à l'identifier politiquement : au-delà des acquis incontestables -que sont notamment la citoyenneté européenne, les valeurs démocratiques (dans les traités) et la protection des droits fondamentaux (dans la Charte)-, quels sont en effet aujourd'hui les fondements spécifiques

${ }^{24}$ P. Soldatos, "Le glissement constitutionnel de la CE/UE vers une gouvernance polyarchique", RMCUE 2011, n 546, pp. 147-155.

25 J.-L. Quermonne, L'Union européenne dans le temps long, op. cit., pp. 179-180.

${ }^{26}$ G. Bossuat, "L'Union européenne, 1990-1997 : une crise du sens", in Construction européenne : crises et relances, op. cit., pp. 51-78.

${ }^{27} \mathrm{Qu}$ 'on en juge (en prenant comme repères la date d'entrée en vigueur d'un traité et la signature du traité suivant) : 4 ans entre Maastricht (novembre 1993) et Amsterdam (octobre 1997), 21 mois entre l'entrée en vigueur de ce traité d'Amsterdam (mai 1999) et la signature du traité de Nice (janvier 2001), 19 mois entre l'entrée en vigueur de ce traité de Nice (février 2003) et la signature du texte de la "Constitution européenne" (octobre 2004).

\section{Crise(s) et droit}


de l'Union ? Or, s'il n'y a pas d'identification, il ne peut y avoir de sentiment d'appartenance, et comme l'affirme un intellectuel "Le problème de l'Europe, c'est d'avoir érodé le sentiment national sans lui substituer pour l'instant un sentiment véritablement européen "28.

Dans ces conditions, il n'est pas véritablement surprenant qu'une proportion importante des référendums organisés ces dernières années sur la construction européenne se soit soldée par des résultats négatifs ${ }^{29}$. Mais ce qui est paradoxal, de prime abord en tous cas, c'est que cette contestation populaire ait mis en échec, à travers la "Constitution européenne", un texte justement porteur d'une véritable ambition politique. L'une des principales explications peut tenir au fait que la logique (plutôt fédérale ?) qui se dégageait de ce texte n'a pas convaincu des populations au sein desquelles le sentiment eurosceptique est devenu dominant, notamment depuis les derniers élargissements : les citoyens européens ont en effet confusément réalisé que ce changement d'échelle avait profondément modifié le sens même de la construction européenne. Et voilà que resurgit le fameux dilemme approfondissement/élargissement...

A cet égard, la multiplication, dans les derniers traités, des exceptions, clauses d'opting out, "flexibilités" et autres coopérations renforcées, si elle a garanti techniquement la poursuite du projet, n'a fait que masquer grossièrement le délitement de la solidarité européenne, "ressuscitée" malgré tout, ponctuellement, comme récemment lors de la crise financière grecque...

Dès lors et pour beaucoup d'observateurs, l'Europe n'a d'autre choix aujourd'hui que d'approfondir sa dimension politique ${ }^{30}$; c'est en renforçant sa consistance politique qu'elle pourra tout à la fois défendre l'euro, agir diplomatiquement sur la scène internationale et décider -enfin- officiellement de ses frontières.

Cette perspective existe bel et bien -même si la voie parait objectivement étroite-, tant l'Europe a fait historiquement la preuve de sa capacité à trouver dans les crises les ferments d'un nouveau départ.

\section{II - LES RÉPONSES AUX CRISES ET LES RELANCES}

Afin de surmonter les crises et d'éviter ainsi l'échec, les Etats membres et les institutions européennes ont dû mobiliser leur énergie, imaginer des compromis, faire des efforts particuliers, concrétisant l'affirmation de Chateaubriand selon laquelle "Les

\footnotetext{
${ }^{28}$ P. BRUCKNER, "Sauver la "vieille dame" de son atonie en revenant à l'esprit des pères fondateurs", Le Monde, 29 mai 2010.

${ }^{29}$ F. Cheneval, "La démocratie et les crises européennes" in Construction européenne : crises et relances, op. cit., pp. 199-217, spéc. p. 205. Pour autant, toues les crises d'opinion ne sont pas forcément des crises de l'Union : D. REYNIE, "Les opinions publiques et les crises de l'Europe", in Construction européenne : crises et relances, op. cit., pp. 79-105, spéc. pp. 99-102.

${ }^{30}$ Voy. J.-L. Quermonne, L'Union européenne dans le temps long, op. cit., pp. 223-228.
}

\section{Crise(s) et droit}


moments de crise produisent un redoublement de vie chez les hommes ${ }^{\prime 31}$. Et les réponses ainsi apportées aux crises ont été juridiques mais aussi politiques (A).

Par ailleurs, les conséquences des crises ont souvent suscité le renouveau, des règles, des mécanismes, voire des comportements ; à cet égard, l'histoire de la construction européenne est rythmée par les enchaînements de crises et de relances qui, globalement considérés, confèrent son dynamisme au projet européen (B).

\section{A - Les réponses, juridiques ou politiques, aux crises}

Eu égard à la place conférée au droit dans le système européen -ce que Walter Hallstein, un autre père de l'Europe, appelait "la puissance du droit"'-, il n'est pas étonnant de constater que les réponses fournies aux crises sont d'abord juridiques; il reste cependant encore une place pour les arrangements politiques ou diplomatiques.

* A l'échelon européen, les "sorties de crise" sont donc, le plus souvent, de nature juridique. La crise permet effectivement de donner naissance à un droit nouveau, plus réaliste, plus adapté à une situation qui n'avait pas été initialement envisagée, bref plus efficace ; diverses modalités sont ici utilisables.

Il y a d'abord la solution législative consistant en l'adoption de règles nouvelles par des actes de droit dérivé (règlements, directives et décisions) ; deux exemples, parmi beaucoup d'autres, permettent d'illustrer cette option.

La fin des années 1990 a été marquée par plusieurs crises sanitaires européennes (listériose, fièvre aphteuse, dioxine, grippe aviaire...), dont la plus connue est assurément celle de l'Encéphalopathie spongiforme bovine plus couramment appelée "maladie de la vache folle",32. Ces crises, qui ont secoué l'Union européenne et mis en évidence de graves dysfonctionnements associés à d'importantes erreurs de gestion au sein de la Commission $^{33}$, ont naturellement conduit à une perte de confiance des consommateurs quant à la capacité de l'Union à gérer les risques sanitaires et alimentaires.

C'est pourquoi, les institutions européennes ont élaboré une législation alimentaire générale reposant sur le règlement 178/2002 du 28 janvier $2002^{34}$, qui prévoyait entre autres nouveautés, la création d'une Autorité européenne de sécurité des aliments (EFSA), plus spécialement chargée d'évaluer les risques "émergents" et de rendre des avis scientifiques destinés d'abord à la Commission européenne.

\footnotetext{
${ }^{31}$ Mémoires d'outre-tombe, 1849-1850.

${ }^{32}$ Pour des précisions sur ces diverses affaires, voy. Y. Pетाт, "Les crises sanitaires et alimentaires", in L'Union européenne et les crises, op. cit., pp. 45-68.

${ }_{33}$ Y. Petit, "Les crises sanitaires et alimentaires", art. préc., p. 47.

${ }^{34}$ Règlement 178/2002/CE du 28 janvier 2002, établissant les principes généraux et les prescriptions générales de la législation alimentaire, instituant l'Autorité européenne de sécurité des aliments et fixant des procédures relatives à la sécurité des denrées alimentaires : JOCE L $31 d u 1^{e r}$ février 2002.
}

\section{Crise(s) et droit}


De même, chacun s'en souvient, en novembre 2002 un navire battant pavillon des Bahamas et vieux de 26 ans, le Prestige, a causé une marée noire au large de la Galice ; cette catastrophe écologique a déclenché une véritable crise politique entre les responsables des pays touchés (Espagne, France et Portugal) et la Commission européenne, chacun se renvoyant la responsabilité de l'existence d'une législation insuffisamment exigeante à l'égard des pétroliers croisant au large des côtes européennes. Tirant les conséquences de cet épisode, les Etats décidèrent donc de modifier le règlement 417/2002 du 18 février 2002 qui prévoyait une généralisation progressive des pétroliers à double coque, afin de renforcer le calendrier initial de retrait des pétroliers à simple coque d'une part et les modalités de contrôle applicables aux "vieux" pétroliers d'autre part ; ce fut fait avec l'adoption, le 22 juillet 2003, du règlement $1726 / 2003^{35}$.

La solution constituante peut également être choisie pour résoudre une crise ; consistant en une modification des traités européens, il s'agit cependant d'une opération à la fois lourde et longue, par ailleurs forcément aléatoire au stade des ratifications nationales.

Ainsi, le "traité modificatif" devenu "traité simplifié" puis "traité de Lisbonne", constitue-t-il la réponse juridique apportée à la crise ouverte par la non-ratification de la "Constitution européenne" au printemps 2005 ; ce texte de "sortie de crise"36 est souvent présenté comme un traité de pure "mécanique institutionnelle", ce qu'il est incontestablement. Pour autant, il convient de souligner qu'il comporte toute une série de mécanismes (généralisation de la "procédure législative ordinaire", principe de l'initiative citoyenne, meilleure information et participation des parlements nationaux au processus législatif...) traduisant clairement la volonté de résorber, au moins partiellement, le déficit démocratique dénoncé depuis longtemps à l'échelon européen ${ }^{37}$ et constituant l'un des facteurs explicatifs de la crise actuelle ${ }^{38}$.

En outre, le principe d'une révision des traités européens a été arrêté fin 2011 afin de mettre en place un dispositif pérenne de gestion des crises financières de la zone euro ; celui-ci a vocation à remplacer le "Fonds européen de stabilité financière" créé lors de la crise grecque en mai 2010 et prévu pour trois ans. Plus précisément, l'ajout d'un paragraphe à l'article 136 TFUE (révisé selon la procédure simplifiée de l'article $18 \S 6$ TFUE) a permis d'instaurer un mécanisme permanent - dénommé "Mécanisme européen de stabilité" (MES) -, lequel prend la forme d'un accord à 26 Etats (le Royaume-Uni

\footnotetext{
${ }^{35}$ Règlement 1726/2003/CE du 22 juillet 2003 : JOUE L 249 du $1^{\text {er }}$ octobre 2003, p. 1.

${ }^{36}$ A. Rigaux, "Derrière les rideaux de fumée du traité de Lisbonne : le "retour" des Etats ?", Mélanges J. Charpentier, Paris, Pedone, 2009, pp. 447-465, spéc. p. 447.

${ }^{37}$ Dans le même ordre d'idée, précisons que la modification des traités européens peut également être ciblée, se limitant à des dérogations accordées à certains Etats afin de passer l'obstacle de la conclusion ou de la ratification : la solution de l'opting out est aujourd'hui connue, tant en matière monétaire (au profit du Danemark, du Royaume-Uni et de la Suède), que dans le cadre du dispositif Schengen ou de l'espace de liberté, de sécurité et de justice (avec la situation particulière de l'Irlande et du Royaume-Uni).

${ }^{38}$ Voy. supra I (B).
}

\section{Crise(s) et droit}


ayant souhaité rester en dehors du système) soumis au processus des ratifications nationales et devant entrer en vigueur en juillet 2012.

En dernier lieu, la solution juridictionnelle peut également s'avérer efficace pour la résolution d'une crise. Dans cette hypothèse, c'est le juge de l'Union qui sera sollicité pour mettre fin à un litige entre les institutions européennes; il agira alors en tant que “juge constitutionnel” faisant respecter la répartition des compétences entre celles-ci.

On rappellera ici que dans les années 1980, il y a eu de vives tensions entre le Parlement et le Conseil des ministres s'agissant de la capacité du premier à saisir la Cour pour qu'elle examine un acte de droit dérivé adopté par le second, alors que rien de tel n'était prévu dans les traités de l'époque. Derrière cette question technique, se cachait évidemment un rapport de force entre une institution incarnant les peuples de l'Union et disposant d'une forte légitimité démocratique et l'autre représentant les Etats. Au nom du principe de "l'équilibre institutionnel", la Cour a admis que le Parlement européen puisse contester devant elle la légalité d'actes "faisant grief" ne formalise cette prérogative $e^{40}$.

Dans la même veine, il faut mentionner rapidement le contentieux se rapportant aux compétences pénales de la Communauté.

Fin 2002, la Commission avait transmis au Conseil un projet de directive relative à la protection de l'environnement par le droit pénal, considérant que même s'il s'agissait de sanctions pénales (relevant normalement du troisième pilier de l'Union à l'époque), celles-ci pouvaient prendre place dans un acte relevant du premier pilier -comprenant la politique de protection de l'environnement-, dans la mesure où elles s'avéraient indispensables pour assurer l'efficacité de l'action européenne en matière environnementale. A l'inverse, le Conseil estimait que l'acte devait être une "décision-cadre" fondée sur une base juridique appartenant au troisième pilier, son contenu étant de nature pénale.

Dès son adoption par le Conseil, le texte -en la forme d'une décision-cadre- fut attaqué par la Commission devant la Cour qui lui donna raison en estimant que même si le droit pénal ne relevait pas de la compétence de la Communauté, l'adoption de sanctions pénales effectives, proportionnées et dissuasives par le législateur européen à l'adresse des Etats, dès lors qu'elle permettait d'assurer l'efficacité de la protection européenne de l'environnement, relevait bel et bien du pilier communautaire ${ }^{41}$. Cette décision fut

${ }^{39}$ C'est ce que l'on appelle la "légitimation active" du Parlement européen (consacrée par l'arrêt CJCE, 22 mai 1990, Parlement c/ Conseil, aff. C-70/88, Rec. I-2041) qui vient compléter la "légitimation passive" -c'est-à-dire la possibilité de contester un acte "faisant grief" adopté par le Parlement européen- consacrée par l'arrêt CJCE, 23 avril 1986, Les Verts c/ Parlement, aff. 24/83, Rec. p. 1339.

${ }^{40}$ Actuel article 263 TFUE (ex-article 230 TCE).

${ }^{41}$ CJCE, 13 septembre 2005, Commission c/ Conseil, aff. 176/03, Rec. I-7879, confirmé par CJCE, 23 octobre 2007, Commission C/ Conseil, aff. C-440/05, Rec. I-9097.

\section{Crise(s) et droit}


d'ailleurs diversement appréciée par les Etats, certains n'hésitant pas à brandir le spectre d'un "gouvernement des juges" ".

Relève enfin de la même logique l'arrêt rendu par la Cour de justice le 13 juillet $2004^{43}$ à propos de la mise en œuvre de la procédure concernant les déficits excessifs prévue au titre du "Pacte de stabilité et de croissance" ${ }^{44}$. Après mûre réflexion, la Commission avait décidé de contester la légalité des décisions prises par le Conseil de ne pas adopter les sanctions proposées par elle à l'encontre de la France d'une part et de l'Allemagne d'autre part pour non respect de la fameuse règle des $3 \%$. En donnant raison à la Commission, le juge mettait juridiquement fin aux vives discussions relatives aux prérogatives respectives des deux institutions dans le cadre de cette procédure de sanction ; mais il permettait également à la Commission de disposer d'une position renforcée dans le débat sur la modification du dispositif qui allait s'ouvrir peu après.

* Dans le cadre européen, la réponse aux crises peut également être de nature politique, démontrant par là-même qu'il y demeure un espace pour la logique diplomatique.

Tel a été le cas avec le compromis de Luxembourg de janvier 1966 qui a permis de mettre fin à la crise de la "chaise vide" ${ }^{45}$; en aucune façon, ce texte ne peut être considéré comme modifiant les règles de vote au sein du Conseil, au point que de nombreux auteurs le qualifient de gentleman's agreement, de déclaration d'intention voire de modus vivendi politique... ${ }^{46}$. Pour autant, il a permis aux Communautés européennes de renouer avec leur fonctionnement normal et il a durablement influencé la prise de décision au sein du Conseil.

La solution politique peut également prendre la forme de l'engagement moral d'une institution à l'égard d'une autre : ainsi, lors de son investiture par le Parlement européen en tant que futur président de la Commission, M. Romano Prodi, tirant les leçons de l'affaire ayant conduit à la démission de la Commission Santer précédemment évoquée, avait pris divers engagements quant au comportement personnel des futurs commissaires européens, engagements formalisés dans un code de conduite transmis aux eurodéputés. Il y avait notamment promis de solliciter l'accord de la majorité du collège pour obtenir la démission individuelle d'un commissaire dans l'hypothèse où celui-ci aurait eu un comportement personnel répréhensible...

${ }^{42}$ Voy. le rapport de l'Assemblée nationale de C. PhilıP n ${ }^{\circ} 2829$ du 25 janvier 2006.

${ }^{43}$ CJCE, 13 juillet 2004, Commission c/ Conseil, aff. C-27/04, Rec. I-6649.

${ }^{44}$ Le Pacte est constitué d'une résolution et surtout de deux règlements organisant d'une part une surveillance multilatérale des politiques budgétaires ainsi qu'économiques, et d'autre part une procédure de sanction en cas de déficit public excessif : règlements $n^{\circ} 1466 / 97$ et $n^{\circ} 1467 / 97$ du 7 juillet 1997 : JOCE L 209 du 2 août 1997, respectivement pp. 1-5 et 6-11.

${ }^{45}$ Le Professeur V. Constantinesco ("Les institutions communautaires et les crises européennes", in Construction européenne : crises et relances, op.cit., pp. 123-140, spéc. p. 135) indique toutefois qu'il avait été un moment envisagé de poursuivre la France pour manquement à ses obligations communautaires du fait de son retrait...

${ }^{46}$ Voy. P. Huberneau, "Le "compromis de Luxembourg" est-il encore d'actualité après l'entrée en vigueur du traité de Lisbonne ?", RMCUE 2010, n 539, pp. 362-367.

\section{Crise(s) et droit}


De même, l'assouplissement du "Pacte de stabilité et de croissance", s'est réalisé, dans la foulée de l'arrêt du 13 juillet 2004 mentionné ci-dessus, par un simple accord politique trouvé en mars 2005, n'entraînant aucune modification formelle des textes de référence.

Enfin, une dernière illustration de l'importance pratique des solutions politiques aux crises européennes est fournie par le plan d'aide à la Grèce décidé dans l'urgence en mai $2010^{47}$. A cette occasion, le montage financier retenu a démontré que la solidarité des Etats de la zone euro l'avait emporté sur le respect des traités ${ }^{48}$, lesquels interdisaient normalement à la BCE et aux banques centrales nationales d'intervenir en faveur d'un Etat en situation financière difficile.

Que sa réponse à la crise soit juridique ou politique, l'Europe a su, jusqu'à présent, systématiquement se relancer.

\section{B - Les relances succédant aux crises}

L'une des constantes bien connues de la construction européenne réside en effet dans cette capacité à rebondir après chaque crise ; comme le note le Professeur Louis Dubouis, depuis l'origine "elle semble progresser par cycles déroulant progrès, enlisement ou crise, puis reprise ",49. Allant même plus loin, certains auteurs ont pu démontrer qu'il existait une véritable "fécondité" des crises dans le processus de construction européenne ${ }^{50}$.

Ainsi, peu après l'échec de la CED, sous l'impulsion des dirigeants des pays du Benelux, le processus européen sera relancé de manière décisive avec comme jalons les conférences de Messine (1955) puis Venise (1956) précédant la signature des deux traités de Rome du 25 mars 1957, instituant respectivement la Communauté européenne de l'énergie atomique (CEEA) et surtout la Communauté économique européenne (CEE). Il est intéressant de noter à cet égard le maintien du choix de la méthode supranationale pour ces nouvelles Communautés (logique pourtant source de blocage du projet de CED peu avant), alors même que l'intégration envisagée à travers la CEE était générale et non plus sectorielle comme dans le cadre de la CECA de 1951.

Par la suite, à la crise de la fin des années 1970 et du début des années 1980 -période marquée notamment par le ralentissement de la croissance économique du fait des chocs pétroliers et l'apparition de certains blocages institutionnels en raison des premiers élargissements des Communautés- a succédé une période particulièrement active entre l'Acte

${ }^{47}$ Voy. Ph. Vigneron, "Réflexions sur le soutien financier à la Grèce. Pour une lecture moins politique du traité", RMCUE 200, n 541, pp. 489-491.

${ }^{48}$ Voy. F. Chaltiel, "Le droit, la crise économique et le pragmatisme européen. A propos du plan d'aide à la Grèce", RMCUE 2010, n 539, pp. 345-348.

${ }^{49}$ L. Dubouis, "Le traité de Lisbonne : traité espéré, traité désespérant ?", in Le traité de Lisbonne. Reconfiguration ou déconstitutionnalisation de l'Union européenne (E. Brosset, C. ChevalierGovers, V. Edjaharian et C. Schneider dir.), Bruxelles, Buylant, 2009.

${ }^{50} \mathrm{H}$. Gelias, "De la fécondité des crises. Le rôle des crises dans la construction européenne", Droits 2007, vol. 45, pp. 35-45.

\section{Crise(s) et droit}


unique européen de 1986 et le traité de Maastricht de 1992 avec pour objectif la réalisation du "Marché intérieur". Certains observateurs n'hésitent pas à la qualifier "d'âge d'or" de la construction européenne, symbolisé tout spécialement par le rôle moteur de la Commission présidée à l'époque par M. Jacques Delors.

Plus près de nous, il est bien évident que l'abandon de la Constitution européenne a ouvert une période de flottement, d'interrogations et même d'immobilisme au sein de l'Union européenne pendant près de dix-huit mois. A partir du Sommet de Berlin début 2007, la relance politico-juridique est pourtant intervenue sous la forme d'un nouveau traité, le traité de Lisbonne. Pour autant, ce rebond n'a été possible que par un processus que d'aucuns ont qualifié de "déconstitutionnalisation" "51 dans la mesure où si l'essentiel du contenu technique de la Constitution européenne a été repris, toutes les dispositions susceptibles de renvoyer à l'image d'un "Etat européen" (principe de primauté, symboles, poste de "Ministre des affaires étrangères de l'Union" notamment) ont été délibérément effacées.

Consacrant pour beaucoup le retour en force des Etats ${ }^{52}$, le traité de Lisbonne doit-il dès lors s'analyser comme une véritable relance de l'intégration européenne ou plutôt comme un rebond en "trompe l'œil" enterrant durablement la progression de l'acquis européen ? La question se pose en effet de savoir, en d'autres termes, s'il s'agit d'une relance réelle ou d'une relance factice.

Seule l'analyse du fonctionnement de l'Union dans les prochaines années permettra d'y répondre ; ce qui est certain en revanche c'est que, comme l'affirmait Jean Monnet, “Les hommes n'acceptent le changement que dans la nécessité et ne voient la nécessité que dans la crise ${ }^{\text {, } 53}$. Dès lors, la situation extrême que connaît la zone euro depuis plus de dix-huit mois pourrait conduire à l'adoption de mesures fortes garantissant une meilleure gouvernance économique entre les Dix-sept, parmi lesquelles la mise en place d'une fédération budgétaire ${ }^{54}$ et la création d'un véritable "gouvernement économique" à partir d'une réorganisation du schéma institutionnel actuel écartelé entre l'Eurogoupe, le Conseil Ecofin, le Conseil européen voire la Commission européenne.

En définitive, il apparaît clairement que la crise a toujours accompagné la construction européenne et continue de l'accompagner. Mais finalement, l'Union connaît-elle aujourd'hui réellement une crise ou n'est-ce pas tout simplement... un déclin ${ }^{55}$ ?

\footnotetext{
${ }^{51}$ Voy. Le traité de Lisbonne. Reconfiguration ou déconstitutionnalisation de l'Union européenne, op.cit.

${ }_{52}$ Voy. J.-L. Quermonne, "Le traité de Lisbonne au regard du traité constitutionnel", in Le traité de Lisbonne. Reconfiguration ou déconstitutionnalisation de l'Union européenne, op. cit., pp. 5-11, spéc. p. 8.

${ }^{53}$ J. Monnet, Mémoires, Paris, Fayard, p. 129.

${ }^{54}$ Le Monde, $1^{\text {er }}$ juin 2010.

${ }^{55}$ G. Toniolo, "L'Europe : crise ou déclin ?", in Construction européenne : crises et relances, op. cit., pp. 165-176. Selon P. BRUCKNER (art. préc.), "l'Europe a incontestablement une histoire mais elle n'est plus l'histoire".
}

\section{Crise(s) et droit}


L'interrogation n'est pas incongrue tant il apparaît que l'Europe a perdu ces dernières années de son influence au niveau international -au profit des puissances émergentes (Inde et Chine tout spécialement)-, qu'elle connaît une période de récession économique, qu'elle a du mal à "absorber" complètement les élargissements de 2004 et 2007, qu'elle présente une légitimité démocratique incomplète, qu'elle n'arrive pas à convaincre les opinions publiques de son efficacité ou encore qu'elle doit faire face à un vieillissement de sa population.

En toute hypothèse, de la situation critique actuelle peut sortir, soit une véritable "déconstruction européenne" signifiant tout simplement la fin de l'aventure européenne, soit une "nouvelle Europe" donnant alors raison à Jean Monnet qui affirmait : "Ai-je assez fait comprendre que la Communauté que nous avons créée n'a pas sa fin en elle-même? La Communauté elle-même n'est qu'une étape vers les formes d'organisation du monde de demain"... 